\title{
Human-Machine Interface for Tele-Robotic Operation: Mapping of Tongue Movements Based on Aural Flow Monitoring
}

\author{
Ravi Vaidyanathan \\ Naval Postgraduate School \\ Monterey, California, USA \\ Lalit Gupta, Beomsu Chung \\ Southern Illinois University \\ Carbondale, Illinois, USA
}

\author{
Thomas J. Allen, Roger D. Quinn, Massood Tabib-Azar, Joseph Zarycki, Joel Levin \\ Case Western Reserve University \\ Cleveland, Ohio, USA
}

\begin{abstract}
A new human-machine interface is introduced for "hands-free" tele-operation of mobile robots. This interface consists of tracking tongue movement by monitoring changes in airflow that occur in the ear canal. Tongue movements within the human oral cavity create unique, subtle pressure signals in the ear that can be processed to produce commands signals in response to that movement. Once recognized, said movements can in turn be used in for robotic tele-operation. The complete strategy is tested on 4 tongue actions: touching the tongue to the left and right corners of the mouth, and to the top and bottom center of the mouth. Through extensive experiments, it is shown that the pressure signals due to tongue movements are distinct and can be detected with over $97 \%$ accuracy. A case study to control the Whegs II robotic platform has specifically been investigated. Based on simulation results, it is concluded that this unique strategy will make hands-free robotic tele-operation a practical reality.
\end{abstract}

Keywords- Human-Machine Interface, Tele-Operation, Pysiological Signals,

\section{INTRODUCTION}

A well-recognized exists for effective tools which will enable smooth tele-opration of robotic platforms. In general, devices for human-machine interface involve detecting an input signal produced by the user and converting that signal into an electronic command that in turn causes a desired event (robotic motion). Presently, the majority of existing systems may be classified as mechanical input devices; i.e. the user physically moves a part of a device in order to generate a control input signal. Examples of such systems include hand-operated joysticks and the tracking of head or chin movements (typically with a lever) whose motion is translated into control commands. Systems of this nature require bodily movement which inherently limits their scope. Furthermore, extended use can cause repetitive motion injuries and skin irritation.
Finally, a single-lever design can limit the range of allowable commands.

Several non-mechanical interfaces have been developed through the use of "biofeedback" to surmount these shortcomings. One example involves measuring the conductivity and electrical sensitivity of the skin, and correlating fluctuations of this measure to operator intent. Since this technique relies on galvanic skin response and electrodermal reflex, nontrivial delays may exist. While an alternative technique of using skin-mounted electrodes can be more responsive, the process of mounting electrodes is cumbersome. Similar devices let users modulate signals by tracking eye movement or ocular activity, which also requires additional sensors. Additionally, all of these techniques have difficulty in producing consistent, repeatable, command signals.

A great deal of recent work has highlighted the potential of the human oral cavity as a source for machine control signals (primarily in rehabilitative applications). Contemporary examples include inserting a track-ball, joystick, plastic palate, or "sip-and-puff" straw into the mouth of an individual with the tongue or lips providing control input. These devices, however, are extremely intrusive, irritate the mouth, impair verbal communication, present hygiene issues, and are also limited in signal generation capacity.

The goal of our work is to develop a human-robotic interface which can overcome the deficits of these systems for seamless tele-operation of mobile platforms. Specifically, we introduce a non-intrusive tonguemovement based machine interface without the need for insertion of any device within the oral cavity. The interface consists of mapping specific tongue motions by monitoring air pressure changes near the human ear and subsequently providing control instruction corresponding to that tongue movement. Our research has shown that various tongue movements create unique, traceable pressure changes in the 
human ear which can be analyzed to produce command and control signals. The system utilizes earpieces similar to contemporary hearing aids. The earpieces contain small passive sensors (off-the-shelf microphones) that detect air pressure changes near the ear whose signatures can be mapped to tongue movement.

The scope of this paper is twofold: 1) to demonstrate that pressure signals in the ear corresponding to tongue movement are distinct, and can be classified and 2) to present a candidate implementation whereby a specific robot may be controlled using this system. As a case study, we have focused on the development of tele-operation strategies specifically applicable to the Whegs II [1] hexapod robot.

\section{TELE-Robotic INTERFACE}

Figure 1A shows a picture of an actual microphoneearpiece housing for pressure signal acquisition next to a picture of a test subject comfortably wearing the device. Figure 1B graphically illustrates the ear-piece/pressure sensor housing inserted into the ear of a test subject. The sensor includes a shielding, housing, and an internal microphone. The internal microphone resides on the interior portion of the housing within the ear canal at a depth of $2.5 \mathrm{~mm}$ to $12.5 \mathrm{~mm}$ measured from the opening of the ear canal. Insertion of the microphone into the ear canal shields pressure signals from environmental noise. The external microphone (not used in this study), will be used in future work to monitor (exclude) external signals.

Figure 2 shows a sample of raw data gathered from a microphone-earpiece (sampled at $2 \mathrm{KHz}$ ) embedded in the housing described earlier, and inserted in the ear of a subject as shown in Figure 1. The subject was asked to lightly move their tongue down and to one side of her mouth, then upwards. As can be seen from the figure, a very clear change in microphone output is seen, which corresponds directly to the movement of the tongue. Not only does the trace offer a very clear indication of the onset of the motion, but its termination is also visibly evident, and nearly all residual traces of the motion are gone after only 0.2 seconds. Thus, a record of the action can be easily identified and subsequent actions taken immediately

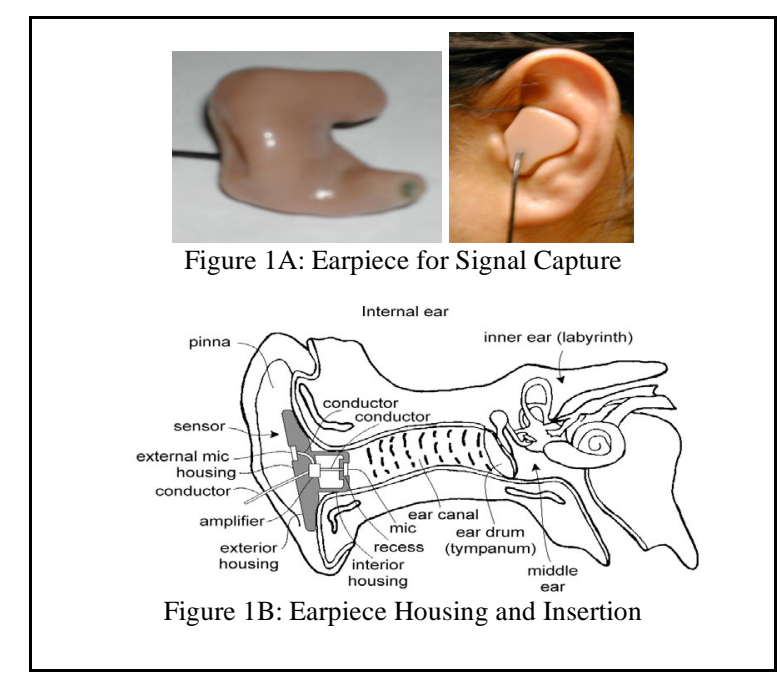

Supported by Air Force contract F08630-01-C-0023, NASA JPL, and NIH grant 1R43HD042367-01A1

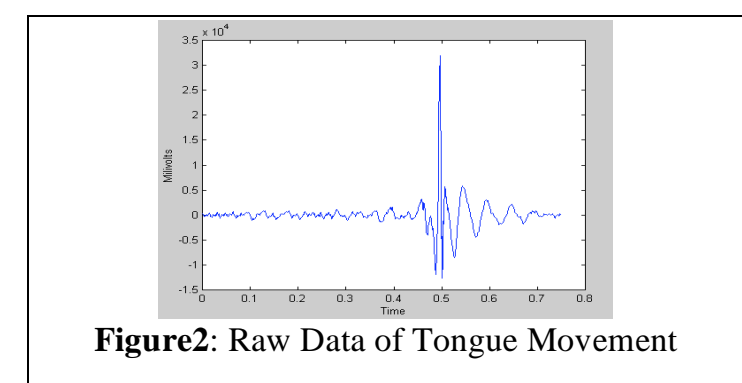

afterward. The short time frame in which the action is mapped allows for very rapid control input.

\section{A. Interface Signal Analysis and Processing}

Conventional signal processing techniques are generally inadequate to recognize the subtle pressure variations in the ear canal resulting from tongue movement. The ear canal itself is an interference-ridden, noiseamplified environment for acoustic recording. External noise (environmental sounds) can also easily obscure the slight pressure deviations accompanying tongue movement. The following two sections enumerate the steps in our current signal processing and classification strategy.

\section{1) Bandpass Filtering and Normalization}

The first step in the analysis of the signals to identify the frequency range of interest in the signals. From our observations, pressure signal activity in the ear from tongue movements is primarily in the band 10 to $50 \mathrm{~Hz}$. Therefore, in the first step of processing, the signals are bandpass filtered using 10 and 50 as the lower and upper cutoff frequencies, respectively. This is a critical to isolate the signal from other physiological actions resulting in pressure waves in the outer ear (speech, for example, has frequency content in excess of $200 \mathrm{hz}$ ).

We have also observed that tongue movement signals typically have amplitude differences within the same class and are not aligned in time. The signals can be easily amplitude normalized by dividing each sample of a signal by the standard deviation of the samples in the signal [2]. In the generalized formulation to follow, let

$$
h_{m, i}(k), k=1,2, \ldots, N_{m} ; i=1,2, \ldots, L
$$

be the $i^{\text {th }}$ filtered and amplitude normalized signal of class:

$$
m, m=1,2, \ldots, M
$$

where, $\mathrm{M}$ is the number of signal classes, $\mathrm{Nm}$ is the number of samples, and $\mathrm{L}$ is the number signals in each class (assumed equal for convenience).

\section{2) Signal Estimation}

Signal averaging is one of the most frequently used operations to estimate signals from the outcomes of a random process [3,4] and can, therefore, be used to estimate the underlying signal of each pressure signal class from the amplitude normalized outcomes. However, directly averaging the signals:

$$
h_{m, i}(k), i=1,2, \ldots L,
$$

will result in a poor time-smeared estimate because the signals are not aligned in time. The accuracy of the estimate can be improved if the signals are first aligned in 
time with a template of each class and then averaged. The problem, however, is that the templates are not available because the true pressure signals are unknown. A pairwise cross-correlation based averaging procedure is introduced to first generate an initial signal template for each class and that can be used to align signals and estimate the signal of each class. If $L$ is assumed to be an integer power of 2 , the average:

$$
\bar{h}_{m, L}(k), m=1,2, \ldots, M
$$

of the $L$ signals can be computed as:

$$
\bar{h}_{m, L}(k)=(1 / 2)\left[\bar{h}_{m ; 1 \rightarrow(L / 2)}(k)+\bar{h}_{m ;(L / 2)+1 \rightarrow L}(k)\right]
$$

where:

$$
\bar{h}_{m ; 1 \rightarrow(L / 2)}(k)=[1 /(L / 2)] \sum_{i=1}^{L / 2} h_{m, i}(k), k=1,2, \ldots N_{m}
$$

is the mean of the first half of the $\mathrm{L}$ signals and:

$$
\bar{h}_{m ;(L / 2)+1 \rightarrow L}(k)=[1 /(L / 2)] \sum_{i=(L / 2)+1}^{L / 2} h_{m, i}(k), k=1,2, \ldots N_{m}
$$

is the mean of the second half of the $L$ signals. By further decomposing the first half and second half of the signals into equally sized sets of size $(L / 4)$, the means can be computed as:

$$
\begin{aligned}
& \bar{h}_{m ; 1 \rightarrow(L / 2)}(k)=(1 / 2)\left[\bar{h}_{m} ; 1 \rightarrow(L / 4)\right.(k) \\
&+\left.\bar{h}_{m ;(L / 4)+1 \rightarrow(L / 2)}(k)\right] \\
& \bar{h}_{m ;(L / 2)+1 \rightarrow L}(k)=(1 / 2)[ \bar{h}_{m ;(L / 2)+1 \rightarrow(3 L / 4)}(k) \\
&\left.+\bar{h}_{m ;(3 L / 4)+1 \rightarrow L}(k)\right]
\end{aligned}
$$

$L$ signals can be decomposed into successively smaller sets until pairs of signals are left. The signals in each pair are averaged by aligning the sequences in the position of maximum cross-correlation. The means of the pairs are combined according to the steps outlined above to determine:

$$
\bar{h}_{m, L}(k), m=1,2, \ldots, M
$$

The initial template for each class is formed by identifying the start- and end-points of the tongue action in:

$$
\bar{h}_{m, L}(k)
$$

and extracting the signal segment between these two points. If the start- and end-points in the initial template are denoted by $a$ and $b$, respectively, each signal

$$
h_{m, i}(k), i=1,2, \ldots L,
$$

is segmented by aligning it with the initial template in the maximum cross-correlation position and multiplying it with a rectangular window:

$$
R_{a, b}(k)
$$

Thus, segmented signals are given by:

$$
h_{m, i}(k) R_{a, b}(k), i=1,2, \ldots, L ; m=1,2, \ldots, M
$$

If $\mathrm{N}=(\mathrm{b}-\mathrm{a}+1)$, the $\mathrm{N}$ samples of the segmented signals are re-ordered and represented by:

$$
v_{m, i}(k), k=1, \ldots, N
$$

The final estimate:

$$
\bar{h}_{m}(k), m=1,2, \ldots, M,
$$

of the signal for each action class can be estimated by averaging the segmented signals:

$$
v_{m, i}(k), k=1, \ldots, N \text {. }
$$

\section{B. Signal Recognition and Classification}

Given the segmented signals belonging to the 4 classes, different classification methodologies can be applied to detect the classes of the signals. We have explored, matched filtering, autoregressive modeling, and non-linear alignment methods to determine the signal classes [5]. Non-linear alignment has shown the greatest promise for recognition accuracy, and is thus enumerated for telerobotic applications.

1) Non-Linear Alignment

Various alignment-based methods can also be formulated to determine the similarity of a test signal and a template of a signal [2,3]. Non-linear alignment, also called dynamic alignment, optimally aligns two signals to compensate for non-linear expansions and compressions in signal segments and for duration differences. In the design of non-linear alignment classifiers, the goal is to determine a mapping $W$ between the time-index $p$ of a test signal $t(p)$ and the time-index $q$ of a reference signal $\bar{h}_{m}(q)$ such that the best alignment between the two sequences is obtained. The mapping

$$
W=[w(1), w(2), \ldots, w(Z)]
$$

where

$$
\begin{gathered}
w(z)=[i(z), j(z)] ; \\
p=i(z), z=1,2, \ldots, Z ; q=j(z), z=1,2, \ldots, Z,
\end{gathered}
$$

defines a piecewise linear alignment path in the $(p, q)$ plane. Both time axes are transformed into a common time axis $\mathrm{z}$ of length $Z$. When there is no timing difference between the sequences, the warping path coincides with the diagonal line $(p=q)$. The best alignment path is given by determining $W$ that minimizes

$$
D=\sum_{z=1}^{Z} d\left[t(i(z)), \bar{h}_{m}(j(z))\right]
$$

where $D$ is the total accumulated distance between $t(p)$ and $\bar{h}_{m}(q)$ along $W$ and $d[x, y]$ is the local distance between the samples $x$ and $y$. Examples of local distance metrics include the absolute difference and the differencesquared norm. In order to restrict $\mathrm{W}$ in a meaningful manner in the (p, q) plane, end-point, continuity, and slope constraints are imposed on $W[2,3]$. If $D_{m}(T)$ is the aligned distance between a test sequence $T$ and a reference sequence

$$
\bar{h}_{m}(q), m=1,2, \ldots, M
$$

then, the test sequence is assigned to the class $\mathrm{m}^{*}$ given by:

$$
m^{*}=\arg \operatorname{MIN}\left[D_{m}(T)\right]
$$




\section{ROBOTIC PLATFORM}

As a case study to demonstrate the utility of the tonguebased human robotic interface, we have designed a candidate control system aimed at directing the Whegs II robot [1]. The Whegs robot is a hybrid wheel/legged platform designed for advanced mobility with minimal energy consumption. Whegs II has a range of passive mechanisms which make it an ideal platform upon which to implement and eventually test a first generation interface mechanism.

\section{A. Whegs Introduction}

Cockroaches have remarkable locomotion abilities. Therefore, one solution to the problem of producing mission capable hexapod robots is to design a robot with the mechanisms responsible for the mobility of a cockroach [7]. In studies of cockroach movement, we have noted the following locomotion principles. A cockroach has six legs, which support and move its body. It typically walks and runs in a tripod gait where the front and rear legs on one side of the body move in phase with the middle leg on the other side. The front legs swing head-high during normal walking so that many obstacles can be surmounted without significant gait changes. However, its gait changes when it encounters larger barriers. The cockroach turns by generating asymmetrical motor activity in legs on either side of its body as they extend during stance [8]. A cockroach enhances its climbing abilities by changing its body postures before and during a climb over an obstacle [9]. It uses its middle legs to pitch its body up prior to climbing obstacles that are higher than its head. This behavior enables its front legs to reach higher. Also, during a climb it uses its body flexion joints to bend the front half of its body down to avoid high centering.

A cockroach enhances its climbing abilities by changing its body postures before and during a climb over an obstacle [9]. It uses its middle legs to pitch its body up prior to climbing obstacles that are higher than its head. This behavior enables its front legs to reach higher. Also, during a climb it uses its body flexion joints to bend the front half of its body down to avoid high centering.

Whegs II (Figure 3) incorporates a body flexion joint in addition to all of the mechanisms that were implemented in its precursor, Whegs I [6]. This new actively controlled joint enables it to perform both of the above posture changes used by the cockroach, thereby improving its climbing ability.

\section{B. Actuation}

Whegs II uses a single DC motor to propel all six of their legs. The former is driven by a hobby $\mathrm{RC}$ car motor through a custom geared transmission. Whegs II is propelled by a $90 \mathrm{~W}$ Maxon motor with an integral transmission, which produces more torque with less frictional losses.

\section{Legs}

A major advantage of legs over wheels is their ability to gain discontinuous footholds, i.e. they alternate between the stance phase, in which they contact the substrate, and the swing phase, in which they do not. This aspect is beneficial on irregular, discontinuous terrain. The Whegs vehicles' three-spoke appendages, called "whegs" (C) R. Quinn, patent pending), abstract the principles of a cockroach's leg cycle while rotating continuously at constant speed.

The Whegs are installed on the vehicles such that they form a tripod gait. The front and rear whegs on one side of the body are in phase with the middle wheg on the opposite side to form a tripod. The two tripods are out-of-phase by 60 degrees. If the vehicle walks in a tripod gait on flat terrain, each spoke will be in stance during only 60 degrees of its rotation. Therefore, even if the spokes were rigid, the hub would translate vertically only about $13 \%$ of the spoke length or body height. This body movement is less than that of an insect during a typical walk. Whegs II uses $10 \mathrm{~cm}$ long spokes that move in the sagittal plane. Each spoke has a spring-loaded prismatic joint, which makes it radially compliant.

\section{Steering}

Whegs II is steered by two small RC servos that are electrically coupled to rotate the front and rear whegs in opposite directions. These rotations alter the direction of ground reaction forces of the feet and cause the robot to change its direction of motion.

\section{E. Compliant Axles for Gait Adaptation}

The tripod gait is not always suitable for a hexapod. In fact, when climbing larger barriers, cockroaches often move their leg pairs in phase. Whegs II has compliant mechanisms in its axles, which accomplish this passively. Inner front, inner middle, and inner rear axles are directly connected to the motor via drive chains. Each inner axle is connected to left and right outer axles via pre-tensioned compliant mechanisms. A large torque on a wheg, during climbing for example, retards the rotation of the wheg. Mechanical stops limit this retardation to 60 degrees, at which point the contralateral wheg has moved into phase with it. These mechanisms cause the robot to run in a nominal tripod gait, but passively adapt its gait to irregular terrain. This compliance captures much of what the cockroach accomplishes with actions of its distal leg joints.

\section{F. Body Flexion}

A cockroach enhances its climbing abilities by changing its body posture before and during a climb over an obstacle [9]. A Whegs vehicle cannot rear up using its middle whegs. However, it can accomplish the goal of raising the front legs higher by rotating a body joint upward.

Whegs II has a body flexion joint that is collocated with its middle axle and is rotated by an RC servo. The front of its body can be flexed up (Figure 3 ) or down 30 degrees. In Figure 3 it is rearing up the front half of its body so that its front legs can reach the top of a step while the rear and middle legs drive it forward. 


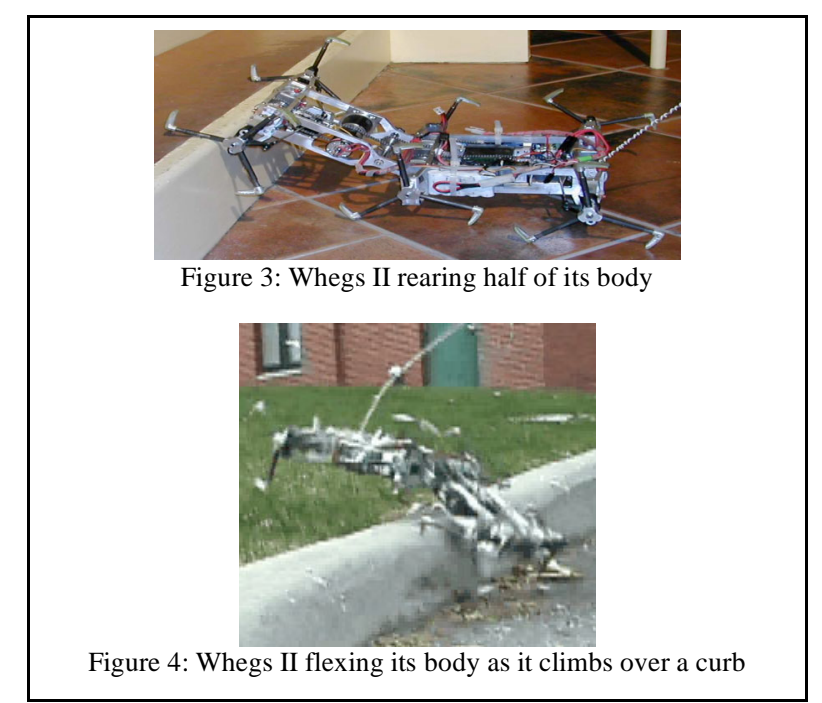

Whegs II's body flexion joint enables the front half of its body to be rotated down, which increases its stability by preventing high centering and permitting the front legs to reach the substrate (Figure 4 ).

\section{G. Summary}

The footprint of Whegs II is $47 \mathrm{~cm}$ long by $36 \mathrm{~cm}$ wide and it weighs $3.86 \mathrm{~kg}$. It has a two-piece aluminium frame and it can flex 30 degrees up and down about its middle axle. It has torsionally compliant devices in all six of its axles. The whegs have internal linear springs $(2280 \mathrm{~N} / \mathrm{m})$ that permit them to comply radially. Its radial wheg-spoke length is $10 \mathrm{~cm}$ when no load is applied. It uses a 90-Watt Maxon motor with a 26:1 integral transmission to propel it, two small hobby servos for steering, and a larger hobby servo to activate the body joint. Its two 7.2 Volt battery packs are placed on its rear body segment such that its center of mass is in the rear and it can lift its front body half. Speed, steering and body joint motion are controlled via a hobby RC system. Whegs II can run at 3 body-lengths per second. Using its body flexion joint, it can readily climb a series of steps (Figure 3) that are 1.38 spokelengths high and 0.8 body-lengths deep. Whegs II can also run as a quadruped on its middle and rear whegs while holding its front whegs airborne.

\section{CONTROL INTERFACE FOR ROBOT (WHEGS II) TELE-OPERATION}

At present, more than 10 distinct repeatable movements of the tongue have been identified as providing a traceable pressure signature which can be captured by the microphone-earpiece housing. The control interface can thus be tailored to any set of movements appropriate to the robot being controlled. In practice, however, larger amounts of movement involve higher levels of complexity and a steeper learning curve for the operator.

We propose a straightforward system designed around four movements for control of the Whegs II platform. These movements, which should be possible for the vast majority of users to execute, include: touching the tongue to the top/front center of the roof of the mouth and lightly "flicking" it forward (top movement), touching the tongue to the bottom/front center of the mouth and lightly "flicking" it up (bottom movement), touching the tongue to the lower right of the mouth and lightly "flicking" it up (right movement), and touching the tongue to the lower left side of the mouth lightly and "flicking" it up (left movement).

These four movements can be coupled to create an intuitive interface such that a right tongue movement corresponds to a right robotic movement, with left movements following naturally. For control, each left/right movement may indicate half a cycle of a sinusoidal input to the steering angle the robot. The robot will thus turn a fixed increment on each left/right input, and subsequently resume a straight path in accordance with the new heading. Repeated movements would increase the direction of the turn. Forward and reverse motions are controlled with top and bottom movements. A top movement inputs a forward velocity signal while a bottom movement results in a backwards velocity input. The forward/backward velocity is altered in a fashion where each additional (top) touch would increase speed by a fixed increment, and a bottom touch would decrease speed by a fixed increment.

Beyond forward/reverse and left/right motions, additional commands may be necessary to control the robot. In order to correlate robot actions to additional movements, a time signature between movements is proposed. In this system, any movement repeated within a fixed time period $(\Delta \mathrm{t})$ of the same preceding movement may be considered to be a separate movement. For example, a top movement followed by another top movement within a period $\mathrm{t}<\Delta \mathrm{t}$ will correlate to a different action than a top movement followed by a pause $>\Delta t$, and another top movement. Based on present data and user feedback, setting $\Delta \mathrm{t}=\sim 0.3$ seconds is comfortable to most users. Note that $\Delta \mathrm{t}$ may be smaller if more rapid control is required; it is relatively easy for a user to repeat the same movement within a very short time frame.

Three inputs beyond motion commands are necessary to complete the Whegs II control interface. In order for the robot to navigate harsh terrain, the operator must be able to specify "flex up" and "flex down" commands to the body flexion joint, and, of course, an "all stop" command to stop robot motion. In the proposed interface, two immediate bottom movements execute an all stop command, while bottom/top and top/bottom tongue motions correlate to up and down body flexion respectively. All enumerated commands would be very straightforward to implement in a standard RC communication setup. Finally, it is very important to note that the tongue movements to be used are very gentle, and will not tire the user any more than speech.

\section{EXPERIMENTS AND RESULTS}

Pressure data corresponding to tongue movements was collected and analyzed to design and evaluate the interface. Each movement was repeated 100 times; therefore, each tongue movement class had 100 pressure signals. Figure 5 shows pressure signals in the ear (again sampled at $2 \mathrm{KHz}$ ), when an operator was asked to execute left, right, top, and bottom movements respectively. Each movement was repeated 100 times, thus each figure has 100 superimposed 
signals corresponding to the same tongue movement. Figure 6 shows the average power spectrum domain (PSD) of each signal shown in Figure 5. Figure 7 shows estimates of the signals of the 4 action classes computed with $L=64$.

Each signal was bandpass filtered and segmented $(\mathrm{N}=$ 800) as described in Section II. The signals were randomly partitioned into 2 mutually exclusive and equal-sized sets to generate a design set and a test set for each class. For each signal class, the signal estimated from the training set was used as the reference template for non-linear alignment The random resampling approach described in $[1,3]$ was used to generate $\mathbf{J}$ design and test set pairs. Each pair is referred to as a trial and the classification accuracies were estimated over $\mathbf{J}=100$ trials. Each trial consisted of testing 50 test signals from each class, therefore, the classification accuracy was estimated with $(100 \times 50 \times 4)=20,000$ signals.

For convenience, the 4 pressure signal classes: left, right, top, and bottom, are represented by $\mathrm{m}=1,2,3$, and 4 , respectively. The non-linear alignment classification results are presented in Table 1 . The table shows confusion matrices as well as the classification accuracies. The confusion matrix part of the results can be interpreted by examining the second row of Table 1 which shows that out of the 5000 tests conducted with signals vectors drawn from class $2,94.94 \%$ were classified correctly as belonging to class $2,1.01 \%$ were misclassified as class 1 , and $4.04 \%$ were misclassified as class 4 . The results show that an overall classification accuracy of $97.73 \%$ is achieved by the non-linear alignment classifier.

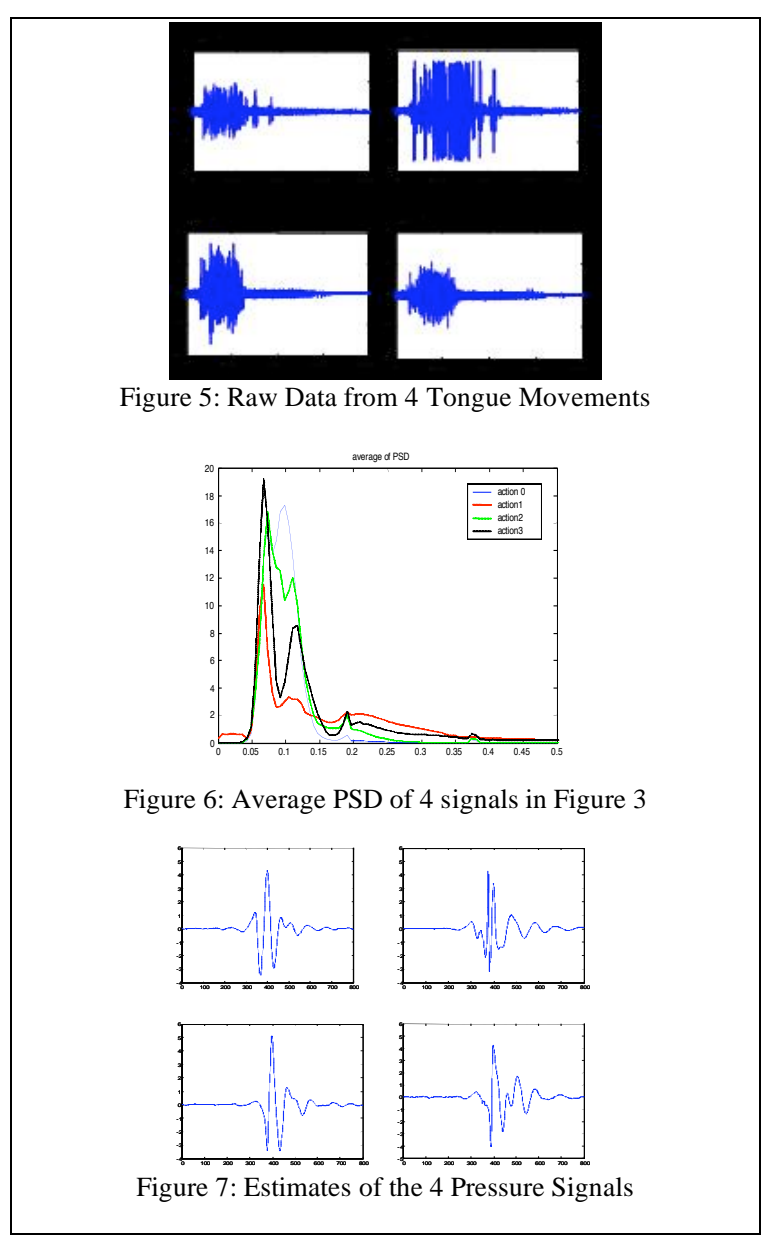

TABLE 1: INTERFACE RECOGNITION ACCURACY

\begin{tabular}{|c|c|c|c|c|}
\hline $\mathbf{m}$ & $\mathbf{1}$ & $\mathbf{2}$ & $\mathbf{3}$ & $\mathbf{4}$ \\
\hline $\mathbf{1}$ & 100 & 0 & 0 & 0 \\
\hline $\mathbf{2}$ & 1.01 & 94.94 & 0 & 4.04 \\
\hline $\mathbf{3}$ & 0 & 0 & 97.98 & 2.02 \\
\hline $\mathbf{4}$ & 1.01 & 0 & 1.01 & 97.97 \\
\hline \multicolumn{6}{|c|}{ Class. Accuracy $=97.73 \%$} \\
\hline
\end{tabular}

\section{RobOtic PERFORMANCE SimUlation}

A simplified version of the control interface has been implemented to control the Whegs II robot in simulation to prove the veracity of this method for robot tele-operation. For the purposes of simulation, the Whegs II robot was assumed to be performing waypoint navigation in an open environment. The assumed task of the interface was to direct the robot to maneuver from its starting point to each of a series of waypoints, stop briefly at that waypoint (presumably for some type of information transfer), then maneuver to the next waypoint. The Whegs II robot was assumed to be capable of maintaining a forward velocity of $1 \mathrm{~m} / \mathrm{s}$ and altering its heading by $5^{\circ}$ movements either to the left or right.

A simplified control interface was implemented for the simulation. In this interface, a "top" tongue movement was assigned to move the robot forward. A "bottom" movement stopped the robot if given when the robot was moving forward. If passed to a stationary robot, this same command would move the robot in reverse. Intuitively, a "right" or "left" movement altered the robot's heading by $5^{\circ}$ in either direction respectively. Tongue movement recognition errors were included based on the accuracies presented in Table 1. For example, if a "right" tongue movement was performed, a $97.98 \%$ probability of the robot receiving this signal was assumed, with a $2.02 \%$ probability of the robot receiving a "top" (forward movement) signal instead. Our work [5] has shown an 0.2 second interval typifies nearly all recorded tongue movements, thus this delay was assumed between movement commands.

Figure 8 shows the results of a simple simulation where the interface was implemented to direct the robot to reach a series of (20) waypoints in a planar work space. The "+" symbols represent the waypoints with the path of the robot shown. The waypoints were spaced arbitrarily across a $200 \mathrm{~m}$ amplitude sinusoidal path with a period of $80 \mathrm{~m}$. In each case, a "virtual" operator was provided the planar position of each successive waypoint and the robot's position. The operator then maneuvered the robot towards the next waypoint based on the 4 previously discussed tongue movements. A waypoint was considered reached if the robot successfully executed a stop within $20 \mathrm{~cm}$ of that waypoint. It should be noted that the location of the next waypoint was withheld from the virtual operator until the current waypoint had been reached. As can be seen from the figure, the robot successfully reached all 20 waypoints. In order to assess the impact of any erroneous operator commands, this particular simulation was repeated 1000 


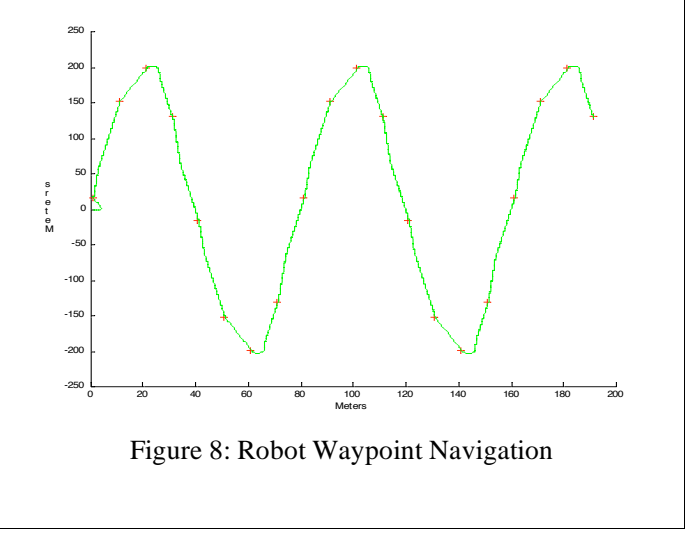

times. In every case, the robot successfully reached all waypoint without fail. An average of 103 commands were mistaken by the interface over each run, but the high rate of accuracy and speed at which commands may be given allowed immediate correction for any mistaken commands.

We consider these results to be highly encouraging for the development of seamless "hands-free" interfaces for robot tele-operation. Current work is focused on implementing higher fidelity command schemes for full tele-operation of the robotic platform in real-time.

\section{ACKNOWLEDGMENT}

Support from Air Force contract F08630-01-C-0023, NASA JPL, and NIH grant 1R43HD042367-01A1

\section{REFERENCES}

[1] Allen, T, Quinn, R.D., Bachmann, R.J., Ritzmann, R.E. “Abstracted biological principles applied with reduced actuation improve mobility of legged vehicles", (2003) Proceedings of the 2003 IEEE International Conference on Intelligent Robots and Systems (IROS)

[2] . L. Gupta and S. Ma, "Gesture-based interaction and communication: automated classification of hand gesture contours," (2001) IEEE Transactions on Sytems, Man, \& Cybernetics - C, vol. 31, No. 1, 114-120, 2001.

[3] L. Gupta, D. L. Molfese, R. Tammana, and P. G. Simos, "Nonlinear alignment and averaging for estimating the evoked potential," (1996) IEEE Transactions on Biomedical Engineering, vol. 43, No. 4, 348-356, 1996.

[4] L. Gupta, J. Phegley, and D.L. Molfese, "Parametric classification of multichannel averaged event-related potentials," (2002) IEEE Transactions on Biomedical Engineering, Vol. 49, No. 8, 905-911, 2002.

[5] Vaidyanathan, R., Kook, H., Gupta. L., West, J., (2004) "Parametric and nonparametric signal analysis for mapping airflow in the ear canal to tongue movement", Paper accepted for publication at the IEEE 2004 International Conference on Acoustics, Speech, and Signal Processing (ICASSP)

[6] .Quinn, R.D., Kingsley, D.A., Offi, J.T. and Ritzmann, R.E., (2002), "Improved mobility through abstracted biological principles", IEEE Int. Conf. On Intelligent Robots and Systems (IROS'02), Lausanne, Switzerland

[7] Ritzmann, R.E., Rice, C.M., Pollack, A.J., Ridgel, A.L. Kingsley, D.A. and Quinn, R.D. (2001) "Roles of descending control in locomotion through complex terrain". Congress of Neuroethology. 6, pg. 234.

[8] Watson, J.T., Ritzmann, R.E., Zill, S.N., Pollack, A.J. (2002) "Control of obstacle climbing in the cockroach, Blaberus discoidalis: I. Kinematics,” J. Comp. Physiology Vol. 188: 39-53.

[9] Yoneda, K. (2001). "Design of non-bio-mimetic walker with fewer actuators. Proceedings of 4th Int. Conf. On Climbing and Walking Robots (CLAWAR), From Biology to Industrial Applications", edited by K. Berns and R. Dillmann, Professional Engineering Publishing, pp. 115-126. 